\title{
Power reduction and resonance avoidance of Maglev vertical axis wind turbines using attractive type passive magnetic bearings
}

\author{
Mahmoud S. MAHMOUD,**, Satoshi UENO* and Changan JIANG* \\ * Department of Mechanical Engineering, College of Science and Engineering, Ritsumeikan University \\ 1-1-1 Nojihigashi, Kusatsu, Shiga, 525-8511, Japan \\ E-mail: sueno@se.ritsumei.ac.jp \\ ** Department of Mechanical Engineering, College of Engineering, Helwan University \\ Ain Helwan, Cairo, Egypt
}

Received: 27 January 2020; Revised: 24 April 2020; Accepted: 24 August 2020

\begin{abstract}
In this paper, a new model of magnetically levitated (Maglev) vertical axis wind turbines (VAWTs) is presented for power generation purposes. The rotor is suspended by two permanent magnet attractive type passive magnetic bearings and one control coil; it is possible to rotate the rotor without any mechanical contact. The proposed model solves the most common problems which are found on the other Maglev VAWTs such as reducing the power consumption during the levitation to zero amperes by the zero-power control method and reducing rotation loss during rotation. In addition, the model has some main advantages consisting in the ability to avoid the resonance at the critical speed and increase the maximum rotation speed by changing the air gap between the rotor and passive magnetic bearings to adjust the radial stiffness of the rotor. The design of the passive magnetic bearings is investigated by the finite element analysis, and the optimum shape is discussed. The results of levitation tests are presented for the model to show the effectiveness of levitation current reduction and the adjustment of radial stiffness. The results of rotation tests show that the resonance can be avoided by changing the radial stiffness which also makes it possible to rotate the rotor over the critical speed. Moreover, the results of free-run tests show that the rotation loss of the proposed Maglev system is quite low.
\end{abstract}

Keywords : Magnetic levitation, Vertical axis wind turbines, Permanent magnet attractive type passive magnetic bearings, Zero-power axial position control, Resonance avoidance

\section{Introduction}

Nowadays, renewable energy sources are becoming increasingly popular as it represents a cheap and environmentalfriendly alternative to traditional energy sources ( $\mathrm{Wu}$ et al., 2009). Wind energy is considered as one of the renewable energy sources that is rich, available in many countries and has great potential in the development of many countries (Renewables Global Status Report, 2019). Low wind speeds tend to be found in areas around the equatorial regions. A wind speed of at least $5 \mathrm{~m} / \mathrm{s}$ is needed for a typical horizontal axis wind turbine (HAWT) to operate and produce energy (Arifujjaman, 2011). Another issue is that these areas are subjected to unstable multi-directional wind which is the cause of making HAWT incompatible in these regions. On the other hand, a vertical axis wind turbine (VAWT) can rotate at low wind speeds and capture wind from different directions, making it more feasible for implementation. Another significant benefit of VAWT is that it can be installed in a place tightly together to allow each turbine counter to rotate in relation to each other. This allows the turbine to rotate in the same direction; thus enhancing the general efficiency of the turbine (Sai et al., 2014). Traditional types of wind turbines that use mechanical bearings and gearboxes have some drawbacks such as mechanical friction between the bearing and the rotor and incapability to operate if the wind speed is not sufficiently high (Cao et al., 2012). As a consequence, it is essential to reduce the starting torque to begin operating the wind turbine at low speeds and to improve the use of wind energy (Wu et al., 2010). 
The implementation of magnetic levitation (Maglev) is an innovative design method for VAWT, which is capable of handling low wind speeds (Shuqin, 2011). A Maglev wind turbine has been designed to eliminate mechanical friction between the rotor and the stator by replacing the conventional mechanical bearing with a magnetic bearing (Wu et al., 2010). In addition, Maglev offers high reliability, easy maintenance, compact size and decreased energy loss (Chiba et al., 2005; Patel and Uddin, 2012). Another study carried out effectively decreases HAWT damping using Maglev design resulting in stable rotation at low wind speeds (Wu et al., 2010; Patel and Uddin, 2012). Even though most Maglev VAWTs use a direct drive generator to avoid mechanical contact, it still requires a large generator diameter to improve the output and efficiency because the rotational speed of the VAWT is low. While all the research on the Maglev HAWTs and VAWTs have been performed, most Maglev wind turbines still use a complex structure with a high-cost design. However, the small wind turbine with low-cost design and simple structure has not reached yet to the practical stage (Bukala et al., 2016).

Some researchers developed a model of Maglev VAWT by using a combination of radial and axial magnetic bearings; however, the structure is complex, and this model needs a high wind speed to start-up and generating electricity (Shuqin, 2011). Although another group of researchers tried to simplify the design and solve the high starting-up wind speed problem by using a pair of the permanent magnet (PM) (repulsive type) to levitate the rotor because it has a simple structure, this structure has certain disadvantages such as cogging torque of the generator, the pull force impact and no stability in the radial direction (Aravind et al., 2012). Therefore, this model had large vibrations during rotation. In addition, both models could not be operated at different wind speeds because they are not capable of adjusting the stiffness of the rotor during the rotation to avoid the resonance at critical speeds. The impacts of merging both Maglev and VAWT to operate it at different wind speeds, which represent high-fluctuated forces that should be absorbed to keep Maglev working stably and safely in both axial and radial direction, remain uninvestigated.

To solve the above problems, a novel VAWT has been introduced (Mahmoud et al., 2018). The rotor shaft is supported by PM attractive type passive magnetic bearings (PMA-PMBs) and an axial position control coil, which realizes noncontact suspension of the rotor with a simple structure and control system. We confirmed that the rotor could levitate and rotate without mechanical contact by the proposed VAWT. Next, we investigated the effect of changing the edge shape of the rotor on improving radial stability (Mahmoud et al., 2019).

In this paper, we introduce a zero-power controller to reduce the power consumption of the Maglev system (Morishita and Azukizawa, 1988). Moreover, the radial stiffness is controlled by changing the air gap between the shaft and PMs, which provides the capability to rotate up over the critical speed of the rotor and reduces the amplitude of vibration (Mori et al., 2019; Ramaratnam and Jalili, 2006).

The remainder of this paper is organized as follows: In Section 2, the proposed model of VAWT is explained in detail including the simple model of a PMA-PMB, zero-power control method, and dynamic model of the rotor. Section 3 analyzes the axial and radial stiffness of the PMA-PMB with various shapes of a shaft edge, and discusses the optimum shape to stabilize both axial and radial directions. The whole design of the prototype including the control system is described in Section 4, and the experimental results are shown in Section 5. The results show that the proposed model can be levitated and rotated without any mechanical contact with quite low power, and the rotor is managed to be rotated over the critical speed by changing the radial stiffness of the rotor.

\section{Maglev system}

\subsection{Whole structure}

In this research, PMA-PMBs are adopted for the suspension of the rotor. A PMA-PMB consists of a PM and an iron shaft. As a result, the structure becomes quite simple, allowing the easy design of the whole rotor including the sensor target, the blades and the generator system. The radial stiffness of the PMA-PMB can be adjusted by changing the air gap length between the shaft and the PM; consequently, it is possible to change the critical speeds of the rotor and to rotate over the critical speeds. Also, the rotational loss can be reduced because of the thin rotor diameter which can reduce the brake torque caused by eddy current.

Figure 1 shows the schematic drawing of the proposed Maglev system for a VAWT. The PMA-PMBs are located over and under the shaft. Because the PMA-PMB is unstable in the axial direction, an axial position control coil and axial displacement sensor are installed. In addition, a gap control device is installed to the lower PM to change the air gap between the PMs and the shaft. An axial flux type generator is installed to generate electricity. 


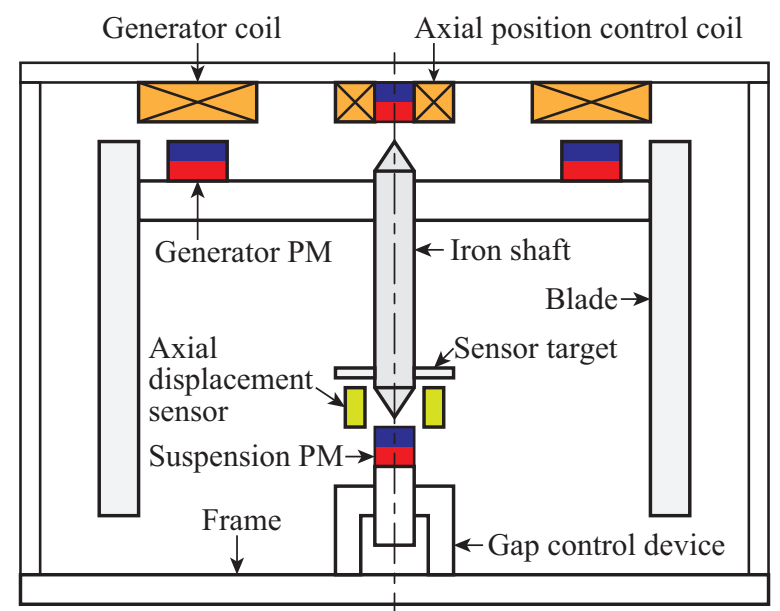

Fig. 1 Schematic drawing of VAWT using Maglev system. Two PMA-PMBs are located over and under the shaft. An axial position control coil is attached to the upper PM to stabilize the rotor shaft. The lower PM can move vertically to change the air gap between the PM and the shaft.

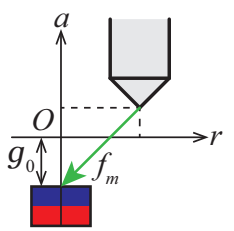

(a)

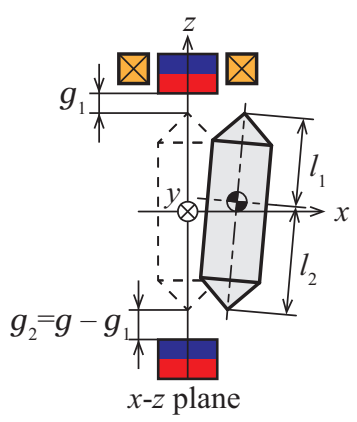

(b)

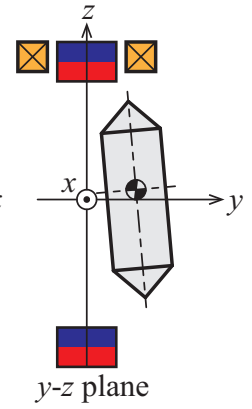

$y-z$ plane

Fig. 2 (a) shows the coordinate system of PMA-PMB. The position of the tip of the shaft is represented by $r$ and $a$. $r$ indicates radial displacement, and $a$ indicates vertical displacement. The PM is located under the origin with an air gap $g_{0}$. (b) shows the coordinate system of the Maglev system. The COG position of the rotor is represented by $x, y$ and $z$, and the rotation around each axis are $\theta_{x}, \theta_{y}$ and $\theta_{z}$. The rotation follows the right-hand rule.

\subsection{Model of PMA-PMB}

We assume that the magnetic attractive force between a PM and a shaft is inversely proportional to the square of the air gap length, and the direction is from the edge of the shaft to the center of the PM. Figure 2 (a) shows the coordinate system of a PMA-PMB, $r$ and $a$ show radial and axial displacements, respectively. The attractive force $f_{m}$ is represented by

$$
f_{m}=\frac{k_{m}}{\left\{g_{\mathrm{PM}}+\sqrt{r^{2}+\left(g_{0}+a\right)^{2}}\right\}^{2}}
$$

where $k_{m}$ is the coefficient of magnetic force, $g_{0}$ is the air gap at $r=0$ and $a=0$, and $g_{\mathrm{PM}}$ is an equivalent air gap length in the PM and shaft. $r$ - and $a$-components of the attractive force are calculated as

$$
\begin{aligned}
& f_{r}=-f_{m} \frac{r}{\sqrt{r^{2}+\left(g_{0}+a\right)^{2}}} \\
& f_{a}=-f_{m} \frac{g_{0}+a}{\sqrt{r^{2}+\left(g_{0}+a\right)^{2}}}
\end{aligned}
$$

By using Taylor expansion at $r=0$ and $a=0$, we have

$$
\begin{aligned}
& f_{r} \approx-\frac{k_{m}}{g_{0}\left(g_{\mathrm{PM}}+g_{0}\right)^{2}} r \equiv k_{r} r \\
& f_{a} \approx-\frac{k_{m}}{\left(g_{\mathrm{PM}}+g_{0}\right)^{2}}+\frac{2 k_{m}}{\left(g_{\mathrm{PM}}+g_{0}\right)^{3}} a \equiv-f_{a 0}+k_{a} a
\end{aligned}
$$


where $f_{a 0}$ is the attractive force at the equilibrium position, and $k_{r}$ and $k_{a}$ are force coefficient with respect $r$ and $a$, respectively. The direction of the radial force is opposite to the displacement, then we obtain restoring force. On the other hand, the direction of the axial force variation is the same as the displacement, then the axial motion becomes unstable. The ratio of the coefficients is calculated as

$$
\frac{k_{r}}{k_{a}}=\frac{1}{2}\left(1+\frac{g_{\mathrm{PM}}}{g_{0}}\right)
$$

This indicates that a smaller air gap produces larger radial stiffness; however, the instability in axial direction becomes severe.

\subsection{Coordinate system of maglev system}

The coordinate system of the Maglev system is defined as Fig. 2 (b). The center of gravity (COG) position of the rotor is represented by $x, y$, and $z$, and the rotation around each axis are $\theta_{x}, \theta_{y}$, and $\theta_{z}$. The rotational direction follows the right-hand rule. $l_{1}$ and $l_{2}$ are the distance between the COG and upper and lower edges of the shaft, and $g_{1}$ and $g_{2}$ are the air gap between the edge of shaft and PM at $x=y=z=0$. The sum of $g_{1}$ and $g_{2}$ is defined as $g$.

\subsection{Axial position control}

Firstly, the axial position control is discussed. Only the axial motion of the rotor is considered. Since the magnetic flux is the sum of flux generated by PM and coil current, the force generated by the coil current is represented by

$$
f_{c}(t)=k_{i} i(t)
$$

where $k_{i}$ is a force coefficient and $i(t)$ is coil current. $k_{i}$ becomes the function of $g$. Thus, the equation of motion of the rotor becomes

$$
m \ddot{z}(t)=f_{z 1}+k_{z 1} z(t)-f_{z 2}+k_{z 2} z(t)-m g+k_{i} i(t)
$$

where $m$ is the mass of the rotor, $g$ is gravitational acceleration and $f_{z 1}, f_{z 2}, k_{z 1}$ and $k_{z 2}$ represent attractive forces and force coefficients at air gap $g_{1}$ and $g_{2}$, respectively. To suspend the rotor mass by only PM force, the equilibrium position is adjusted to satisfy the following relationship.

$$
f_{z 1}-f_{z 2}=\frac{k_{m}}{\left(g_{P M}+g_{1}\right)^{2}}-\frac{k_{m}}{\left(g_{P M}+g-g_{1}\right)^{2}}=m g
$$

Accordingly, the equation of motion becomes

$$
m \ddot{z}(t)=\left(k_{z 1}+k_{z 2}\right) z(t)+k_{i} i(t) \equiv k_{z} z(t)+k_{i} i(t)
$$

where $k_{z}$ is the stiffness due to the two PMA-AMBs. $k_{z}$ becomes a function of $g$ because the equilibrium position is determined from Eq. (9). The transfer function from the control current to displacement, $P(s)$ becomes

$$
P(s)=\frac{Z(s)}{I(s)}=\frac{k_{i}}{m s^{2}-k_{z}}
$$

where $Z(s)$ and $I(s)$ are Laplace transformation of $z(t)$ and $i(t)$, respectively.

To reduce power consumption, we adopt the zero-power controller (Morishita and Azukizawa, 1988). This control method achieves the reduction of the power in which the attractive force produced by the permanent magnets is balanced by the weight of the floated object, and the control current converges to zero. A distinctive feature of the zero-power

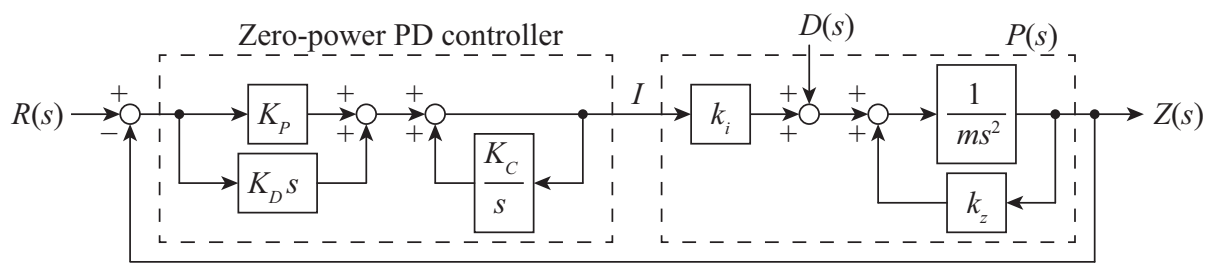

Fig. 3 Block diagram of the closed-loop system of the MAGLEV system. The zero-power PD controller consists of a PD controller and local integral feedback of the current $I(s)$. The control current $I(s)$ converges to zero due to the local integral feedback loop despite step inputs to reference $R(s)$ and disturbance $D(s)$. 
control is that it has negative stiffness (Mizuno and Takemori, 2002; Hoque et al., 2006). In this research, a zero-power control method is used to control the axial position of the rotor and reduce the current during the levitation. The zeropower controller consists of a proportional-derivative (PD) controller and local positive integral feedback. The block diagram is shown in Fig. 3, where $K_{P}, K_{D}$, and $K_{C}$ are a proportional gain, derivative gain, and local integral feedback gain, respectively, $R(s)$ is the reference position, and $D(s)$ is disturbance caused by the imbalance of Eq. (9) or any external disturbance factors. When the step inputs are applied to reference $(R(s)=R / s)$ and disturbance $(D(s)=D / s)$, the current becomes

$$
I(s)=\frac{\left(m s^{2}+k_{z}\right)\left(K_{P}+K_{D} s\right) R-\left(K_{P}+K_{D} s\right) D}{m s^{3}+\left(k_{i} K_{D}-m K_{C}\right) s^{2}+\left(k_{i} K_{P}-k_{z}\right) s+k_{z} K_{C}}
$$

Therefore, the coil current converges to zero when $t \rightarrow \infty$ despite of $R$ and $D$.

\subsection{Radial motion and resonance avoidance}

The state-space equation in radial motions without disturbance is expressed as

$$
\left[\begin{array}{c}
\dot{x} \\
\dot{y} \\
\dot{\theta}_{y} \\
\dot{\theta}_{x} \\
\ddot{x} \\
\ddot{y} \\
\ddot{\theta}_{y} \\
\ddot{\theta}_{x}
\end{array}\right]=\left[\begin{array}{cccccccc}
0 & 0 & 0 & 0 & 1 & 0 & 0 & 0 \\
0 & 0 & 0 & 0 & 0 & 1 & 0 & 0 \\
0 & 0 & 0 & 0 & 0 & 0 & 1 & 0 \\
0 & 0 & 0 & 0 & 0 & 0 & 0 & 1 \\
-\alpha / m & 0 & 0 & \beta / m & 0 & 0 & 0 & 0 \\
0 & -\alpha / m & -\beta / m & 0 & 0 & 0 & 0 & 0 \\
0 & \beta / J & -\gamma / J & 0 & 0 & 0 & 0 & -J_{p} \Omega / J \\
-\beta / J & 0 & 0 & -\gamma / J & 0 & 0 & J_{p} \Omega / J & 0
\end{array}\right]\left[\begin{array}{c}
x \\
y \\
\theta_{y} \\
\theta_{x} \\
\dot{x} \\
\dot{y} \\
\dot{\theta}_{y} \\
\dot{\theta}_{x}
\end{array}\right]
$$

where $\alpha, \beta$ and $\gamma$ are represented by

$$
\begin{aligned}
& \alpha=k_{r 1}+k_{r 2} \\
& \beta=k_{r 1} l_{1}-k_{r 2} l_{2} \\
& \gamma=k_{r 1} l_{1}^{2}+k_{r 2} l_{2}^{2}
\end{aligned}
$$

$\Omega$ is rotation angular velocity, $J$ is radial moment of inertia, $J_{p}$ is polar moment of inertia and $k_{r 1}$ and $k_{r 2}$ are radial stiffness of upper and lower PMA-PMBs, respectively. The natural frequencies are calculated by eigenvalue analysis of the above matrix. When $\Omega=0$, natural angular frequencies $\omega_{1}$ and $\omega_{2}$ are calculated as

$$
\begin{array}{lll}
\omega_{1}=\sqrt{\frac{\alpha}{2 m}+\frac{\gamma}{2 J}-\sqrt{\left(\frac{\alpha}{2 m}-\frac{\gamma}{2 J}\right)^{2}-\frac{\beta^{2}}{2 m J}}} & {[\mathrm{rad} / \mathrm{s}]} \\
\omega_{2}=\sqrt{\frac{\alpha}{2 m}+\frac{\gamma}{2 J}+\sqrt{\left(\frac{\alpha}{2 m}-\frac{\gamma}{2 J}\right)^{2}-\frac{\beta^{2}}{2 m J}}} & {[\mathrm{rad} / \mathrm{s}]}
\end{array}
$$

The natural frequencies depend on the radial stiffness of the PMA-PMBs; therefore, they can be changed by adjusting the air gap between the shaft and PMs.

To avoid the resonance at $\omega_{1}$ caused by the unbalance of the rotor, the natural frequency is switched between high and low angular frequencies denoted by $\omega_{1 h}$ and $\omega_{1 l}$. When $\Omega \ll \omega_{1 h}$, the resonance can be avoided by setting the natural frequency to $\omega_{1 h}$. When $\Omega \approx\left(\omega_{1 h}+\omega_{1 l}\right) / 2$ during acceleration, the resonance at $\omega_{1 h}$ can be avoided by switching the natural frequency from $\omega_{1 h}$ to $\omega_{1 l}$.

\section{Design of PMA-PMB}

In a PMA-PMB, the shaft edge can have various shapes. As mentioned in Section 2, the smaller air gap increases the radial stiffness; however, the difficulty of controlling the axial motion increases as well. In this research, we compared five shapes by finite element analysis (FEA) (Mahmoud et al., 2019). Figure 4 (a) shows the analyzed shapes of the rotor edge; F is flat, $\mathrm{S}$ is hemisphere, C-I, C-II, and C-III are cones with an apex angle of $120^{\circ}, 90^{\circ}$, and $60^{\circ}$, respectively.

Three-dimensional, nonlinear, static electromagnetic analyses were conducted by JMAG Designer 17.1 (JSOL), which is a simulation software for the development and design of electrical devices (JMAG-Designer, 2019). A neodymium (NdFe-B) magnet is adopted for the PM, and soft magnetic iron (SUY-1) is adopted for the shaft. 


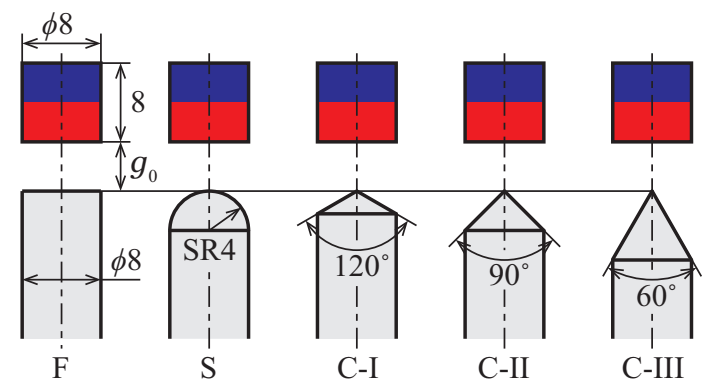

(a)

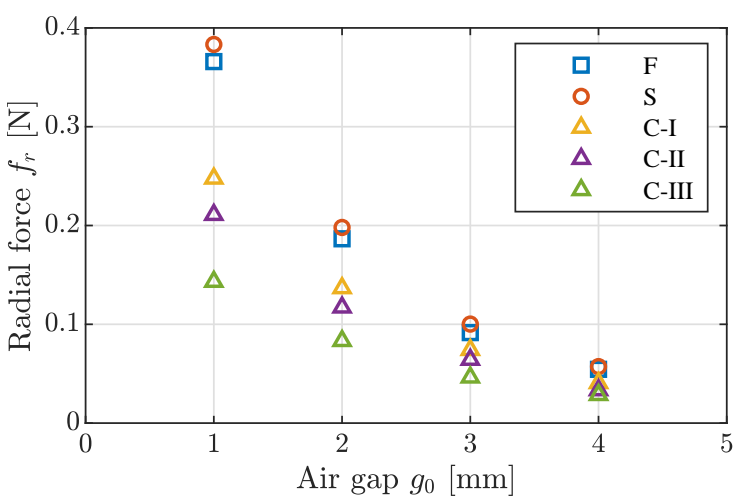

(c)

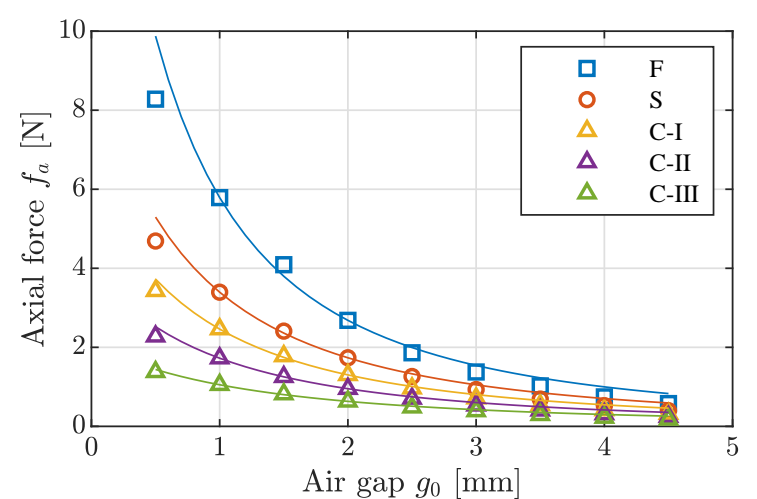

(b)

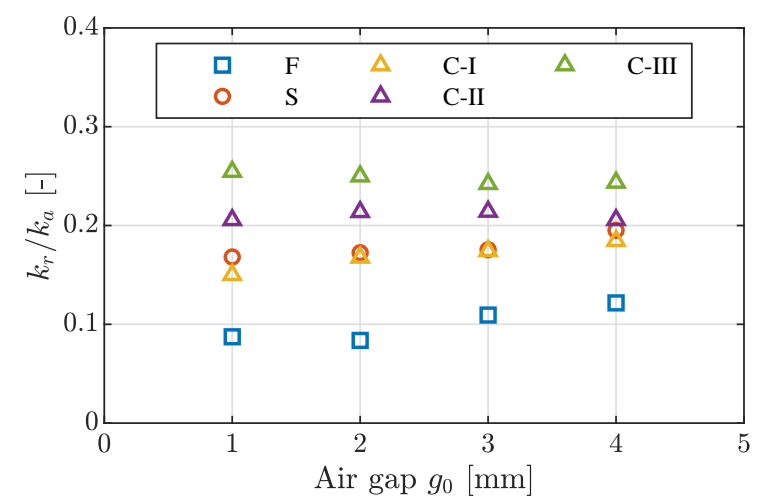

(d)

Fig. 4 (a) shows the shapes of rotor edge; F is flat, $\mathrm{S}$ is a hemisphere, C-I, C-II, and C-III are cones with an apex angle of $120^{\circ}, 90^{\circ}$, and $60^{\circ}$. (b) shows the FEA results of axial force with respect to the air gap between the shaft and PM. The axial force is inversely proportional to the square of the air gap. (c) shows the FEA results of radial force at radial displacement of $1 \mathrm{~mm}$. The shape $\mathrm{F}$ has the largest radial force, while the shape C-III has the smallest at the same air gap length. However, the shape C-III has the largest radial force for the same axial force. (d) shows the ratio of the radial stiffness and the axial stiffness at various air gaps. The shape C-III has the largest ratio, which means this shape has the largest radial stiffness at the same axial force or the same axial negative stiffness.

The relation between the air gap and the attractive force is shown in Fig. 4 (b). The axial force is inversely proportional to the square of the air gap as shown in Eq. (5). The shape F, flat edge, has the largest axial force; consequently, the negative stiffness becomes the largest. On the other hand, the shape C-III, conical edge with the apex angle of $60^{\circ}$, has the smallest force and thereby the smallest negative stiffness. The axial force and negative stiffness are affected by the shape of the shaft edge.

The radial forces at the radial displacement of $1 \mathrm{~mm}$ are shown in Fig. 4 (c). The results were different from Eq. (4) because the magnetic flux passes through not only the apex of the shaft but also the surface of the edge. The shape also affects the radial force, and the shape $\mathrm{F}$ has the largest radial force, while the shape $\mathrm{C}$-III has the smallest value at the same air gap length. However, for the same axial force, the shape C-III has the largest radial force, while the shape $\mathrm{F}$ has the smallest value.

Figure 4 (d) shows the ratio of the radial stiffness and the axial stiffness at various air gaps. The radial and axial stiffnesses are calculated from the results of Figs. 4 (b) and (c). This result shows that the shape C-III has the largest ratio, which means that this shape has the largest radial stiffness at the same axial force or the same axial negative stiffness. When the axial negative stiffness increases, a high proportional gain is required for stable levitation; however, it is difficult to realize a high gain due to noise, lag and current limit in the control system. It is desirable small axial negative stiffness for axial position control whereas large radial stiffness is required for stable rotation. We, therefore, adopted the shape C-III for the experimental device.

\section{Test Machine}

Figure 5 (a) shows the photograph of the experimental device. The device consists of a rotor, two PMA-PMBs, an axial position control coil and six sensors. The PMs of PMA-PMBs are fixed to XYZ stages to change the air gap $g$. The 


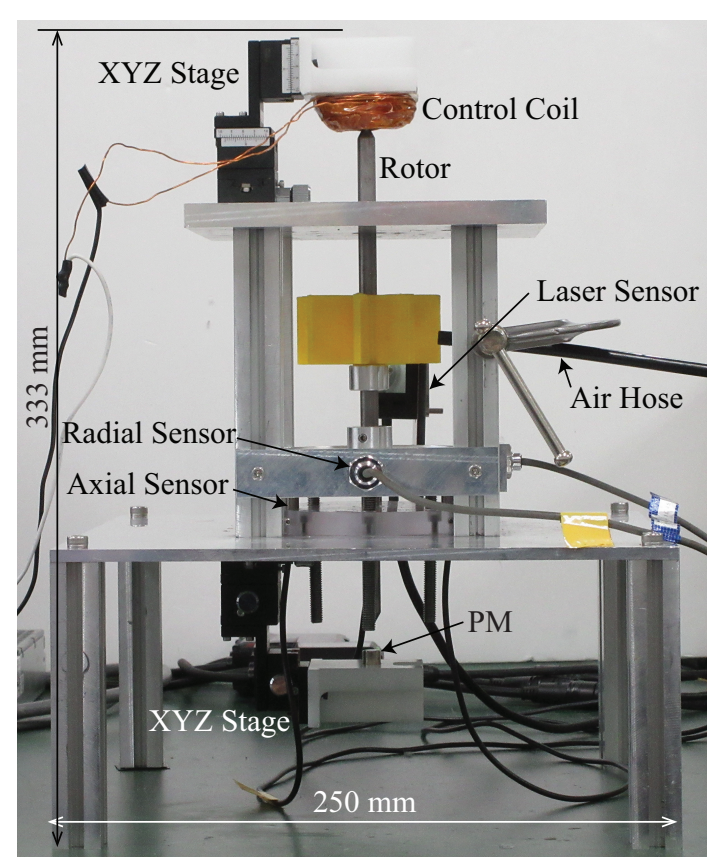

(a)

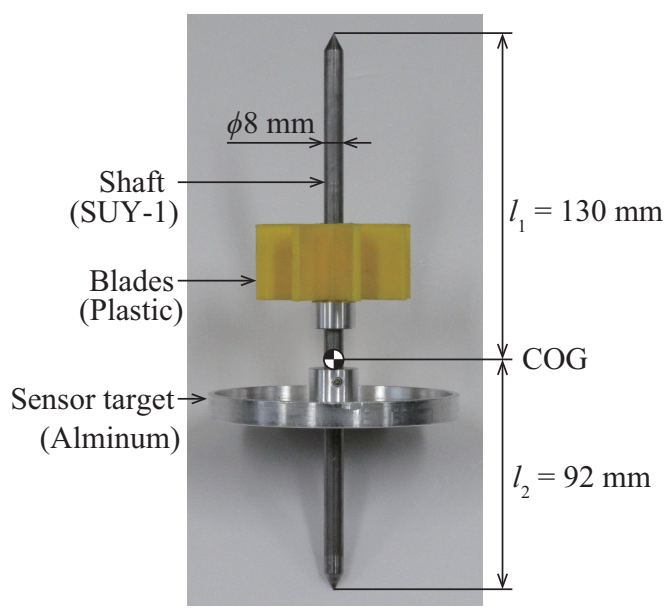

(b)

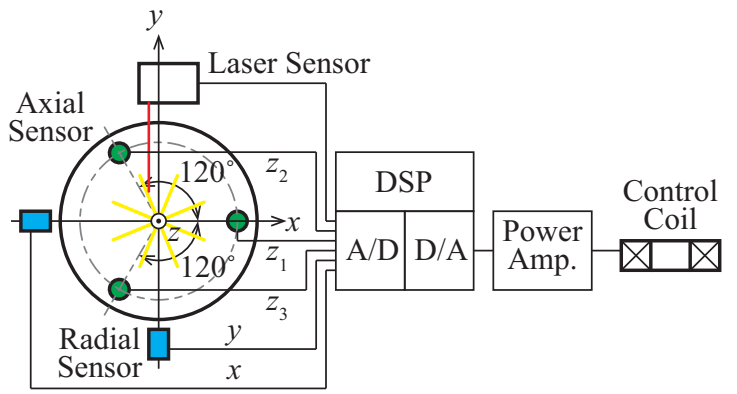

(c)

Fig. 5 (a) shows the photograph of the experimental device. The device consists of a rotor, two PMA-PMBs, an axial position control coil and six sensors. (b) shows the photograph of the rotor. The rotor consists of a shaft, a sensor target and blades which are composed of eight plates. The size of the plate is $30 \mathrm{~mm}$ hight, $25 \mathrm{~mm}$ width and $3 \mathrm{~mm}$ thickness. (c) shows the control system. The axial position is actively controlled while the other axes are only monitored.

control coil is wounded directly around the upper PM. The specifications of the control coil are shown in Table 1.

Three eddy current displacement sensors (OMRON, ZX-ED02) are installed on the bottom of the sensor target to measure the axial displacement and tilt angles of the rotor, and two eddy current displacement sensors (SENTEC, HA101S) are installed on the side of the sensor target to monitor the radial motions. Furthermore, a laser displacement sensor (OMRON, ZX2-LD50) is installed on the side of the blades to measure the rotation speed. The rotation speed is measured by the period of the pulse from the laser sensor. An air compressor is used for blowing air to rotate the rotor.

Figure 5 (b) shows the photograph of the rotor. The rotor consists of a shaft, a sensor target and blades. Table 2 shows the specifications of the rotor. The moments of inertia were measured by a bifilar pendulum method.

Figure 5 (c) shows a control system. A digital signal processor (DSP, dSPACE, DS1104) is used. The DSP reads the signals from the sensors via analog to digital (A/D) converters, and calculates the coil current by the zero-power controller. The axial displacement is obtained from the average of three sensors, and the zero position is defined at the position where the rotor contacted the upper PM. The current command is sent to a power amplifier (Copley, Junus JSP-090-10, Voltage: $24 \mathrm{~V}$, Current: $\pm 5 \mathrm{~A}$, Bandwidth: $2.7 \mathrm{kHz}$ ) via a digital to analog (D/A) converter. The gains of the controller were experimentally determined by trial and error as shown in Table 3. An approximate differentiator is used instead of $s$ to avoid the influence of noise.

\section{Experimental results}

\subsection{Start-up tests}

Firstly, we conducted start-up tests at different $g$. Figure 6 (a) shows the axial position $z$ and control current $i$ during the start-up tests. At $t=0$, the position control was turned on, then the current became $-2.0 \mathrm{~A}$ immediately. In cases of $g=2.4 \mathrm{~mm}$ and $2.7 \mathrm{~mm}$, the rotor moved immediately, and converged to equilibrium positions. In other cases, the rotor stayed at the initial position after starting the control, then the amplitude of the current increased gradually due to current integral feedback. When the attractive force of the upper PM was canceled by the attractive force of the lower PM and control current, the rotor pulled away and moved to equilibrium positions. These results confirm that the stable 
Table 1 Specifications of the control coil.

\begin{tabular}{ll}
\hline Wire & PEW, $\phi 0.7 \mathrm{~mm}$ \\
Turns & 300 \\
Resistance & $1 \Omega$ \\
Inductance (without shaft) & $370 \mathrm{mH}$ \\
\hline
\end{tabular}

Table 2 Specifications of the rotor.

\begin{tabular}{ll}
\hline Mass $m$ & $0.226 \mathrm{~kg}$ \\
Radial moment of inertia $J$ & $4.7 \times 10^{-4} \mathrm{kgm}^{2}$ \\
Polar moment of inertia $J_{p}$ & $1.6 \times 10^{-4} \mathrm{kgm}^{2}$
\end{tabular}

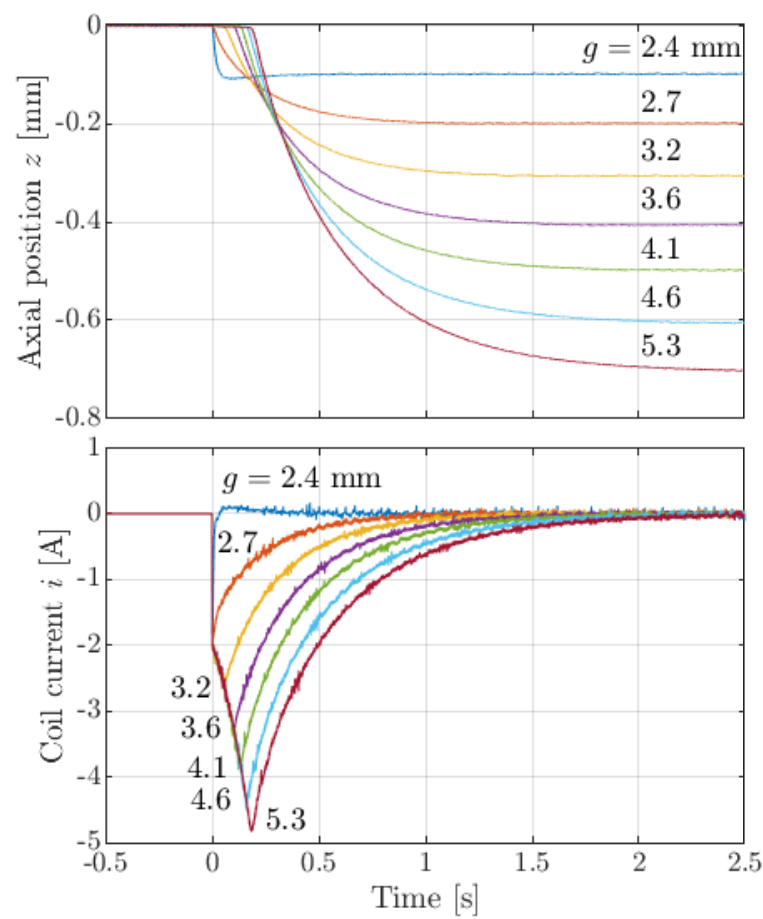

(a)
Table 3 Gains of the controller.

\begin{tabular}{ll}
\hline Proportional gain $K_{P}$ & $20 \mathrm{~A} / \mathrm{mm}$ \\
$\begin{array}{l}\text { Derivative gain } K_{D} \\
\text { Break frequency of approximate }\end{array}$ & $0.2 \mathrm{As} / \mathrm{mm}$ \\
differentiator & $2 \pi \times 100 \mathrm{rad} / \mathrm{s}$ \\
Current integral gain $K_{C}$ & $5 \mathrm{~A} / \mathrm{As}$ \\
$\begin{array}{l}\text { Reference position } R \\
\text { Sampling time }\end{array}$ & $-0.1 \mathrm{~mm}$ \\
Discretization & $0.1 \mathrm{~ms}$ \\
\hline & Tustin method \\
\hline
\end{tabular}

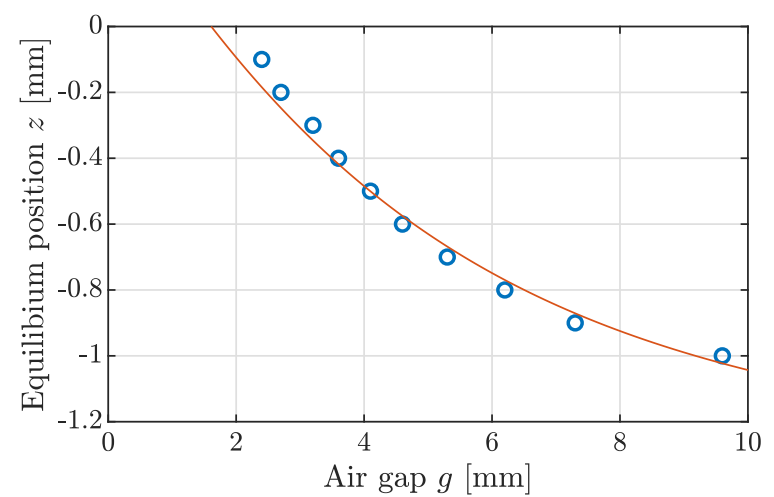

(b)

Fig. 6 (a) shows start-up responses at different air gaps. At $t=0$, the position control was turned on. The rotor moved to the equilibrium positions, and the current converged to zero. (b) shows the relationship between the air gap $g$ and equilibrium position $z$. A regression curve is calculated from Eq. (9) with $k_{m}=56.1 \times 10^{-6} \mathrm{Nm}^{2}$ and $g_{P M}=3.63 \times 10^{-3} \mathrm{~m}$.

levitation and the zero current control were achieved at the different air gaps. The copper loss was reduced from $25 \mathrm{~W}$ at the maximum current $(5 \mathrm{~A})$ to almost $0 \mathrm{~W}$ during the steady-state.

Figure 6 (b) shows the relationship between the air gap $g$ and equilibrium position $z$. A regression curve obtained from Eq. (9) is drawn, and they are in good agreement.

\subsection{Impulse responses in the radial direction}

Next, we measured impulse responses by hitting the upper tip of the shaft during the levitation. Figure 7 (a) shows the results of the impulse response at $2.4 \mathrm{~mm}$ air gap. The upper and lower graphs show the axial position $z$ and tilt angle $\theta_{x}$, respectively. The axial position was well controlled despite the radial vibration. The vibration in the radial displacement and tilt angle remained a long time due to lack of damping.

Figure 7 (b) shows the power spectrum of $\theta_{x}$. The vibration consists of two components. The frequencies of the first and second modes are $7.6 \mathrm{~Hz}$ and $24.2 \mathrm{~Hz}$, respectively. Schematic shapes of each mode are shown in the same figure.

Figure 7 (c) shows the measurement and analytical results of the natural frequencies at various air gaps. Plots are measured values while lines are analytical values. The analytical values were calculated by Eqs. (17) and (18). $k_{r 1}$ and $k_{r 2}$ were determined to fit the first mode frequencies. The natural frequencies were depended on the air gap, and decreased by increasing the air gap. There is a large difference between the measurement and analytical results in the second mode frequencies. One of the reasons for this difference is considered the effect of the tilt angle of the shaft relative to the center 

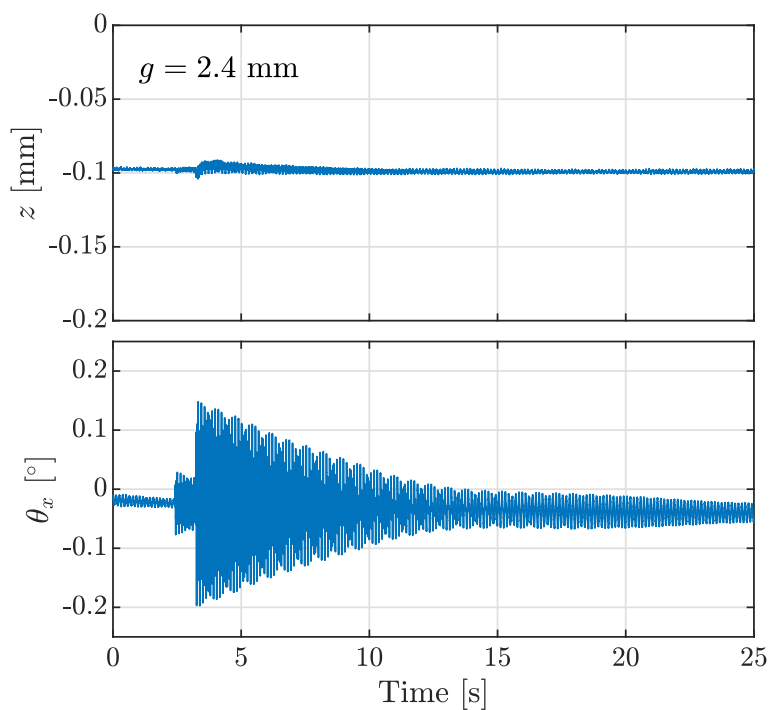

(a)

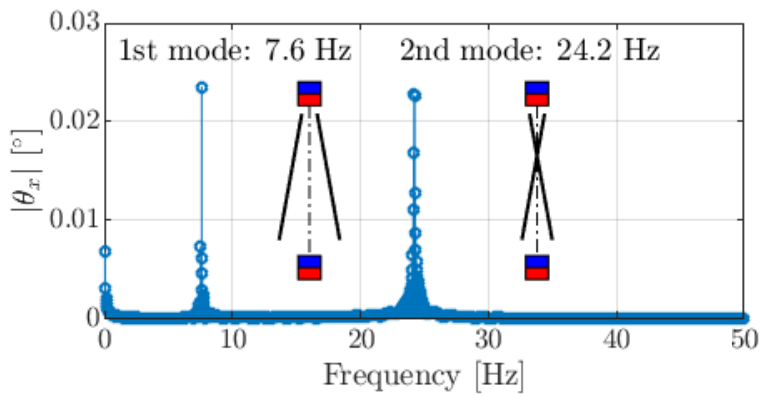

(b)

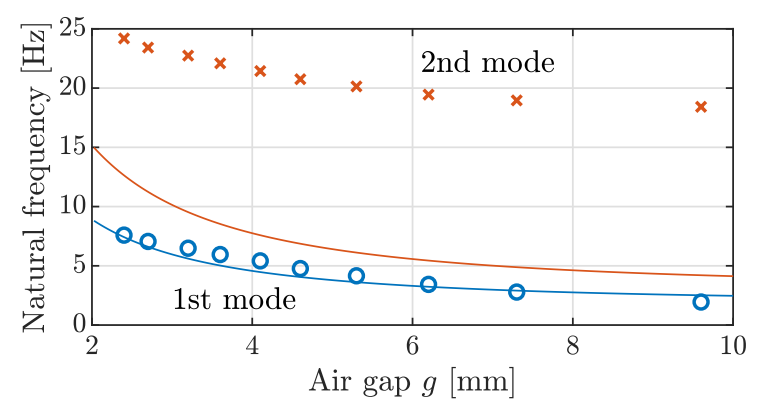

(c)

Fig. 7 (a) shows waveforms of $z$ and $\theta_{x}$ when impulse disturbance was given to the upper tip of the shaft. (b) shows the power spectrums of $\theta_{x}$ and mode shapes at natural frequencies. (c) shows the measurement and analytical results of the natural frequencies at various air gaps.

line of the PM which was not considered in the FEM analysis. Further analysis including the tilt angle is required to derive the precise model.

\subsection{Rotation tests}

Rotation tests were conducted by blowing air to the blades. Figures 8 (a) and (b) show the results of the rotation tests at $2.4 \mathrm{~mm}$ air gap and $4.1 \mathrm{~mm}$ air gap, respectively. The upper, middle, and lower graphs show rotational speed, axial position, and radial displacement, respectively. The maximum speeds were about $440 \mathrm{rpm}$ at $2.4 \mathrm{~mm}$ air gap, and $320 \mathrm{rpm}$ at $4.1 \mathrm{~mm}$ air gap. The vibration increased due to resonance at the first natural frequency $(7.4 \mathrm{~Hz}$ and $5.4 \mathrm{~Hz})$; consequently, the rotor touched the frame, and could not rotate faster.

To avoid the resonance, the air gap was changed during acceleration by hand. Figure 8 (c) shows the result when the air gap was changed from $2.4 \mathrm{~mm}$ to $4.1 \mathrm{~mm}$ at around $400 \mathrm{rpm}$ which is almost the middle of each first natural frequency. Although the vibration increased around the first natural frequency, contact between the rotor and frame was avoided by changing the air gap, and thus the rotor could rotate up to about $620 \mathrm{rpm}$. This result demonstrates that the rotor can rotate over the critical speed by changing the radial stiffness during acceleration.

Figure 8 (d) shows the results of free-running at different air gaps. The graphs indicate rotational speed, axial position and radial displacement from $300 \mathrm{rpm}$. The rates of decreasing speed at the two air gaps were almost the same, and the running time was over 10 minutes. This means that rotation loss is quite small. The radial vibration at $4.1 \mathrm{~mm}$ air gap was larger than that at $2.4 \mathrm{~mm}$ when the speed was over $100 \mathrm{rpm}$ due to the influence of the resonance. The amplitude of the vibrations was almost the same at low rotational speeds. The vibration at low speed was caused by the runout of the sensor target. The amplitude, therefore, was independent of the stiffness of the radial bearings.

\section{Conclusions}

A new model of Maglev VAWT using two PMA-PMBs has been proposed in this study. The axial and radial stiffness of the PMA-PMB were analyzed by FEM, and it was shown that the ratio of radial and axial stiffness was improved by using the conical edge of the shaft. The zero-power controller was adopted for the axial position control, and the experiments showed that the power consumption during the levitation was reduced. The experimental results showed that 
Mahmoud, Ueno and Jiang, Mechanical Engineering Journal, Vol.7, No.5 (2020)

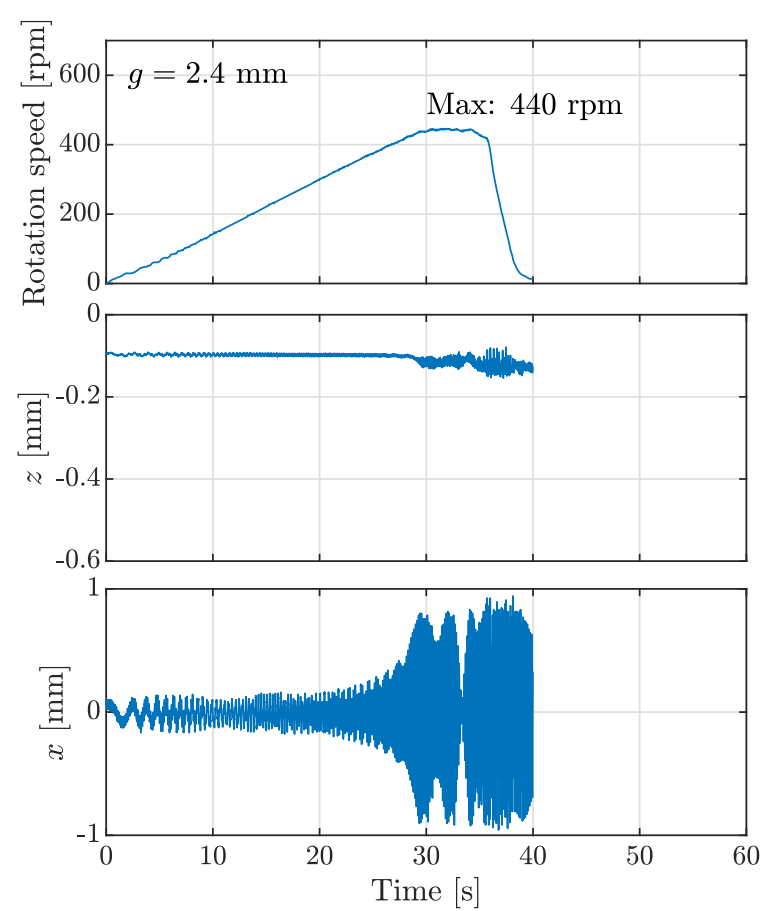

(a)

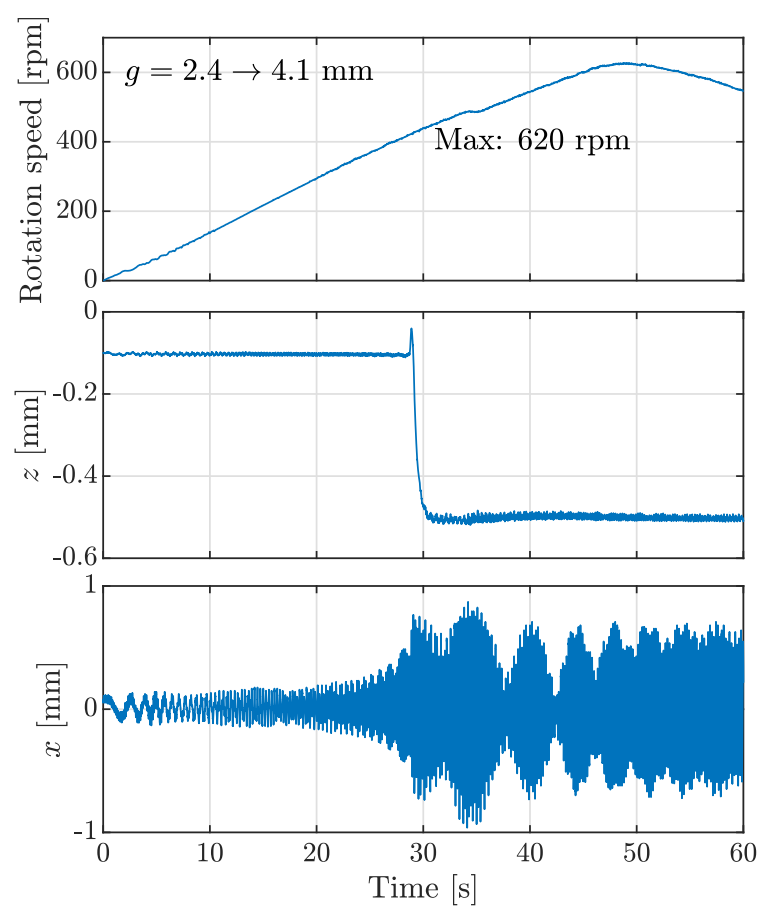

(c)

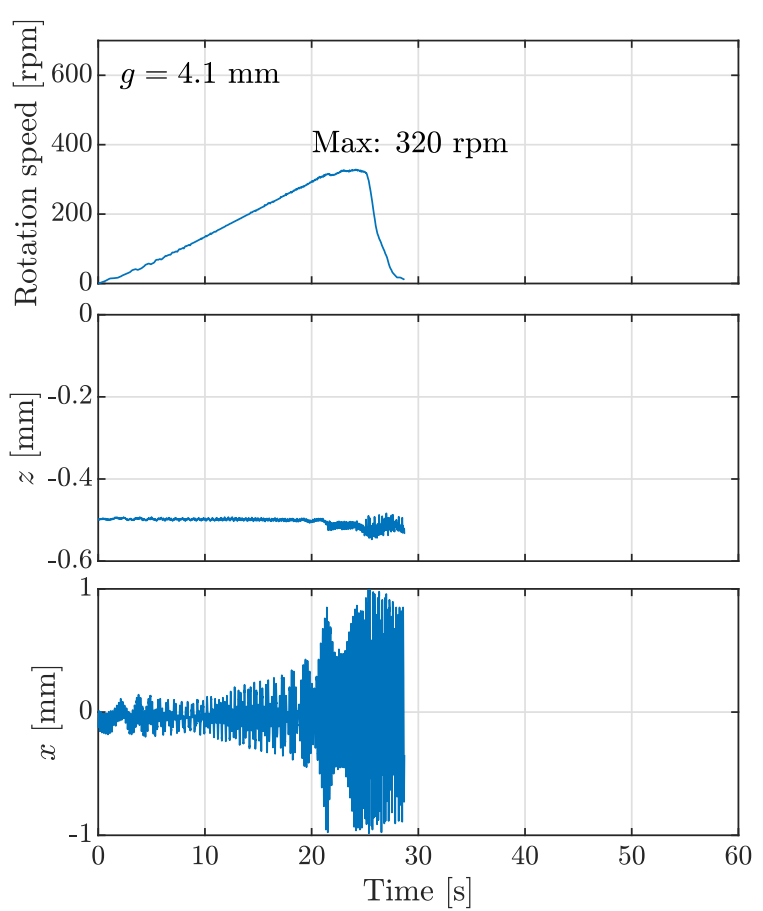

(b)

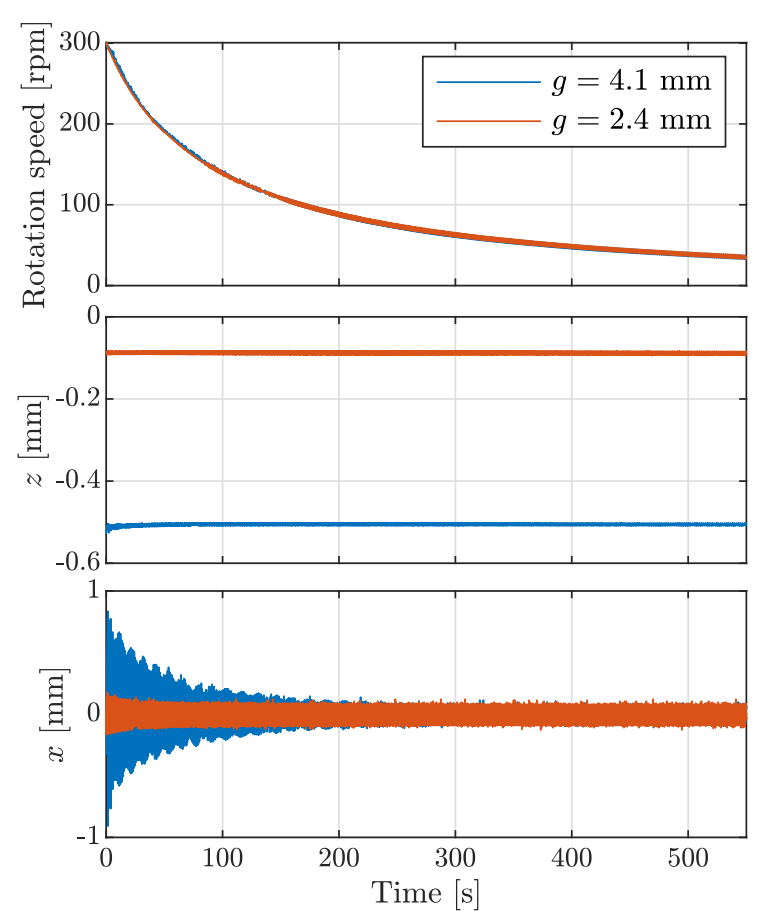

(d)

Fig. 8 Results of rotational tests at (a) $g=2.4 \mathrm{~mm}$, (b) $g=4.1$ and (c) $g=2.4 \rightarrow 4.1 \mathrm{~mm}$. By changing the air gap at almost the middle rotational speed of first natural frequencies of each air gap, the rotor rotated up to over the critical speed. (d) shows the results of free-running tests at two air gaps. In both cases, the rotor rotated for more than 10 minutes. As a result, it can be concluded that the proposed Maglev system has a quite low rotational loss. 
the radial stiffness was controlled by changing the air gap, and it was possible to rotate the rotor over the critical speed by changing the air gap during acceleration. The free-running tests showed that the rotation loss of the PMA-PMB was quite small.

From the above results, we conclude that the proposed Maglev VAWT is suited for the wind turbines because it has a simple structure, low power loss and capability to rotate over the critical speed. In the future, we will introduce a mechanism that automatically controls the air gap.

\section{References}

Aravind, C. V., Rajparthiban, R., Rajprasad, R. and Wong, Y. V., A novel magnetic levitation assisted vertical axis wind turbine - Design procedure and analysis, 2012 IEEE 8th International Colloquium on Signal Processing and its Applications, Melaka (2012), pp. 93-98.

Arifujjaman, M., Modeling and Simulation of Grid Connected Permanent Magnet Generator (PMG)-based Small Wind Energy Conversion Systems, The Open Renewable Energy Journal, vol. 4 (2011), pp. 13-18, .

Bukala, J., Damaziak, K., Kroszczynski, K., Malachowski, J., Szafranski, T., Tomaszewski, M., Karimi, H. R., Jozwik, K., Karczewski, M. and Sobczak, K., Small Wind Turbines: Specification, Design, and Economic Evaluation, Wind Turbines - Design, Control and Applications, IntechOpen (2016).

Cao, W., Xie, Y., and Tan, Z., Wind Turbine Generator Technologies, Advances in Wind Power, IntechOpen, IntechOpen (2012).

Chiba, A., Fukao, T., Ichikawa, O., Oshima, M., Takemoto, M. and Dorrell, D. G., Magnetic bearings and bearingless drives, Newnes (2005).

Hoque, M. E., Takasaki, M., Ishino, Y. and Mizuno, T., Development of a three-axis active vibration isolator using zeropower control, IEEE/ASME Transactions on Mechatronics, vol. 11, no. 4 (2006), pp. $462-470$.

JMAG-Designer (online). available from [https://www.jmag-international.com/products/jmag-designer/]. (accessed: 31Aug-2019).

Mahmoud, M. S., Ueno, S. and Jiang, C., Vertical-Axis Turbines Using Permanent Magnet Attractive Type Passive Magnetic Bearings, The 16th International Symposium on Magnetic Bearings (ISMB16), Beijing, China (2018).

Mahmoud, M. S., Ueno, S. and Jiang, C., Stiffness Analysis of Vertical-Axis Wind Turbines Rotors Using Permanent Magnet Attractive Type Passive Magnetic Bearings, The12th Asian Control Conference (ASCC), Kitakyushu, Japan (2019), pp. 1222-1227.

Mizuno, T. and Takemori, Y., A transfer-function approach to the analysis and design of zero-power controllers for magnetic suspension systems, Electrical Engineering in Japan, Vol. 141, No. 2 (2002), pp. 67-75.

Mori, K., Asama, J., Oiwa, T. and Torii, T., Resonance avoidance for a 2-DOF controlled maglev motor using d-axis current, Transactions of the JSME (in Japanese), Vol. 85, No. 872, (2019), DOI:10.1299/transjsme.18-00417.

Morishita, M. and Azukizawa, T., Zero power control of electromagnetic levitation system, Electrical Engineering in Japan, Vol. 108, No. 3 (1988), pp. 111-120.

Patel, N. and Uddin, M. N., Design and performance analysis of a magnetically levitated vertical axis wind turbine based axial flux PM generator, 2012 7th International Conference on Electrical and Computer Engineering, Dhaka (2012), pp. 741-745.

Ramaratnam, A., and Jalili, N., A switched stiffness approach for structural vibration control: theory and real-time implementation, Journal of Sound and Vibration, Vol. 291 (2006), pp. 258-274.

Renewables Global Status Report - REN21, (online), available: http://www.ren21.net/status-of-renewables/global-statusreport/ (accessed on 14-May-2019).

Sai, P. C., Yadav, R. S., R. Raj, N. and Gupta, G. R. K., Design and simulation of high efficiency counter-rotating Vertical Axis Wind Turbine arrays, 2014 International Conference and Utility Exhibition on Green Energy for Sustainable Development (ICUE), Pattaya (2014), pp. 1-9.

Shuqin, L., Magnetic Suspension and Self-pitch for Vertical-axis Wind Turbines, Fundamental and Advanced Topics in Wind Power, IntechOpen (2011).

Wu, H., Wang, X. and Hu, Y., Development of Small Wind Generator Based on Hybrid Magnetic Bearing, 2009 AsiaPacific Power and Energy Engineering Conference, Wuhan (2009), pp. 1-4.

Wu, H., Wang, Z., and Hu, Y., Study on Magnetic Levitation Wind Turbine for Vertical Type and Low Wind Speed, 2010 Asia-Pacific Power and Energy Engineering Conference, Chengdu (2010), pp. 1-4. 